\title{
Carboplatin and vincristine chemotherapy for children with newly diagnosed progressive low-grade gliomas
}

Roger J. Packer, M.D., Joanne Ater, M.D., Jeffrey Allen, M.D., Peter Phillips, M.D., Russell Geyer, M.D., H. Stacy Nicholson, M.D., Regina Jakacki, M.D., Elizabeth Kurczynski, M.D., Michael Needle, M.D., Jonathan Finlay, M.B., Ch.B., Gregory Reaman, M.D., and James M. Boyett, Ph.D.

Departments of Neurology, Pediatrics, and Hematology-Oncology, Children's National Medical Center, George Washington University, Washington, D.C.; Division of Pediatrics, M.D. Anderson Cancer Center, Houston, Texas; Department of Neuro-Oncology, New York University Medical Center, New York, New York; Departments of Neurology and Oncology, Children's Hospital of Philadelphia, University of Pennsylvania, Philadelphia, Pennsylvania; Department of Oncology, Children's Hospital and Medical Center, University of Washington, Seattle, Washington; Department of Oncology, Riley Hospital for Children, Indianapolis, Indiana; Department of Oncology, Scottish Rite Children's Hospital, Atlanta, Georgia; Department of Pediatric Oncology, Memorial Sloan-Kettering Cancer Center, New York, New York; and Department of Biostatistics, St. Jude Children's Research Hospital, Memphis, Tennessee

The optimum treatment of nonresectable low-grade gliomas of childhood remains undecided. There has been increased interest in the use of chemotherapy for young children, but little information concerning the long-term efficacy of such treatment. Seventy-eight children with a mean age of 3 years (range 3 months-16 years) who had newly diagnosed, progressive low-grade gliomas were treated with combined carboplatin and vincristine chemotherapy. The patients were followed for a median of 30 months from diagnosis, with 31 patients followed for more than 3 years. Fifty-eight children had diencephalic tumors, 12 had brainstem gliomas, and three had diffuse leptomeningeal gliomas. Forty-four (56\%) of 78 patients showed an objective response to treatment. Progression-free survival rates were $75 \pm 6 \%$ at 2 years and $68 \pm 7 \%$ at 3 years. There was no statistical difference in progression-free survival rates between children with neurofibromatosis Type 1 and those without the disease (2-year, progression-free survival $79 \pm 11 \%$ vs. $75 \pm 6 \%$, respectively). The histological subtype of the tumor, its location, and its maximum response to chemotherapy did not have an impact on the duration of disease control. The only significant prognostic factor was age: children 5 years old or younger at the time of treatment had a 3-year progression-free survival rate of $74 \pm 7 \%$ compared with a rate of $39 \pm 21 \%$ in older children $(p<0.01)$. Treatment with carboplatin and vincristine is effective, especially in younger children, in controlling newly diagnosed progressive low-grade gliomas.

Key Words * glioma * chiasmatic glioma * low-grade glioma * brainstem tumor * chemotherapy * children

Gliomas comprise more than $50 \%$ of all childhood brain tumors and approximately $80 \%$ of these lesions 
are low grade at the time of diagnosis.[8] The light microscopic features of childhood low-grade gliomas vary significantly. The most common subtypes are pilocytic and fibrillary, although histologically more complex tumors with mixed glial elements and/or ganglionic components (ganglioglioma) frequently occur. $[6,8,13]$ The cerebellum is the most frequent site of origin of childhood low-grade gliomas, but a significant proportion of tumors will arise in other regions of the central nervous system, especially in the diencephalon area in children younger than 5 years of age.

The optimum treatment for childhood low-grade gliomas remains undecided. Total surgical removal, as defined by the surgeon's operative impression and postoperative imaging, results in a high rate of disease control for children with pilocytic cerebellar astrocytomas.[7] For gliomas that arise in other regions of the brain, such as the chiasm, hypothalamus, thalamus, or cerebral cortex, the benefit of aggressive surgery is less well documented.[2,7,9,10,27] Although extensive resections are often possible and may result in at least transient disease control, they may also result in significant neurological and cognitive sequelae.[27]

For those patients in whom total or near-total resection cannot be safely performed, radiation therapy has often been recommended. Radiotherapy may result in objective shrinkage of low-grade gliomas and there is some evidence to indicate that it will increase the duration of progression-free survival in patients with residual tumor after surgery.[3,4,22,23,26] However, there has been significant reluctance to use radiotherapy in very young children. This is because of the vulnerability of the immature nervous system to therapeutic doses of radiation and the tendency of low-grade gliomas, especially in very young children, to infiltrate the central nervous system extensively, which results in the requirement of large radiotherapy volumes. In addition, radiotherapy carries the risk to the patient of developing a second malignant neoplasm years after the completion of the treatment.

The use of radiotherapy is probably even more problematic in children who have neurofibromatosis Type 1 (NF-1).[16] In some series, as many as 15 to $20 \%$ of children with low-grade gliomas have NF-1. Patients with this disease often have multiple abnormal areas of brain near regions of presumed tumor, confounding decisions concerning the volume of brain to be included within the radiation portal. In addition, it has been postulated, but not proven, that nervous system tissue in patients with NF-1 may be more sensitive to the mutagenic effects of radiotherapy. For these reasons, there is even greater reluctance to use radiotherapy in these patients.

Over the past two decades, there have been multiple reports on the efficacy of chemotherapy in children with both newly diagnosed and recurrent low-grade gliomas. The majority of trials have been performed in children with chiasmatic lesions. A variety of drugs have been used, including vincristine, actinomycin $\mathrm{D}$, etoposide, carmustine, and carboplatin, as well as combination regimens consisting of these drugs (R. Prados, personal communication, 1996).[5,14,17,18,20,21,24] In 1989, a study was begun to evaluate the efficacy of combining carboplatin and vincristine in treating children with recurrent and newly diagnosed astrocytomas. The objective response rates of 60 children, including 37 with newly diagnosed astrocytomas, have been reported.[18] However, because of the short length of the follow-up review, this report did not address the primary goals of therapy: the duration of disease stability or improvement and the delay of the need for further types of potentially more toxic treatment. In addition, therapy was initially limited to children 60 months of age or younger with newly diagnosed disease. Since the preliminary publication, the number of newly diagnosed patients who have been treated using this protocol has doubled, older patients have been made eligible, and patients have been followed for a sufficiently long period to address the issue of how long the treatment can be effective. In the present 
report, we review the overall disease control in 78 children with newly diagnosed, progressive low-grade gliomas, each of whom completed all therapy and has been observed for a median of 30 months from the time of diagnosis. We also update the rate of response to, as well as the toxicity of, this drug regimen.

The study was opened in August 1989 and closed to patient accrual in April 1993. Initially, only children 60 months of age or younger with newly diagnosed progressive low-grade gliomas were eligible for treatment. Beginning in June 1991, children between the ages of 61 and 180 months were also made eligible for study. Histological diagnoses deemed eligible for study included low-grade astrocytoma, low-grade oligoastrocytoma, low-grade oligodendroglioma, mixed low-grade glioma, and ganglioglioma. Newly diagnosed patients required histological verification of a low-grade glioma before entry into the study, except for those patients with NF-1 and those with progressive, diffusely infiltrating diencephalic lesions. Patients with gliomas of the optic nerves were only eligible if there was contiguous chiasmatic involvement. The latter groups of patient could be enrolled in the study on the basis of neuroradiographic findings alone, although biopsy was strongly recommended. Patients with lesions in any site of the neuroaxis were eligible for study. Patients with newly diagnosed disease were allowed to be placed on the protocol if they had pretreatment evidence of either radiographic (> 25\% enlargement on sequential neuroimaging studies) and/or clinical deterioration before surgery, and if less than $50 \%$ of the tumor was debulked at the time of surgery. Patients who had received prior radiotherapy or chemotherapy consisting of drugs other than corticosteroids were not eligible.

Prior to their entry into the study, patients were required to undergo either computerized tomography (CT) scanning, with and without addition of contrast agent, or magnetic resonance (MR) imaging, with and without administration of gadolinium. Although CT scanning was allowed by the protocol, all patients, in fact, were diagnosed and followed by MR imaging. Spinal MR imaging and cerebrospinal fluid cytological examination performed prior to enrollment in the study were recommended, but not mandatory.

Patients were required to have radiographically measurable disease before initiation of treatment. The level of response to treatment was determined by investigators at the treating institution. There was no central neuroradiographic review. In addition, the pathological diagnosis was determined at the treating institution.

\section{Treatment Protocol}

Chemotherapy was scheduled to commence within 4 weeks of documentation of tumor progression and/or initial tumor surgery. Treatment began with an induction regimen of carboplatin and vincristine. Carboplatin was administered by a 1-hour infusion at a dose of $175 \mathrm{mg} / \mathrm{m}^{2}$ for 4 consecutive weeks, followed by a 2-week rest period, and then reinstituted for 4 more weeks. Vincristine at a dose of 1.5 $\mathrm{mg} / \mathrm{m}^{2}$ (maximum dose $2 \mathrm{mg}$ ) was administered by intravenous bolus weekly for 10 weeks, concurrent with the carboplatin.

Patients who exhibited an objective radiographic response to induction treatment and at least clinical stability and patients who had stable clinical or neuroradiographic disease were given maintenance chemotherapy. This consisted of four intravenous doses of carboplatin at $175 \mathrm{mg} / \mathrm{m}^{2}$ and vincristine at $1.5 \mathrm{mg} / \mathrm{m}^{2}$ (maximum dose $2 \mathrm{mg}$ ) administered weekly for the first 3 weeks of each 4 -week cycle. There was a 3-week rest between each maintenance cycle and, in children with stable or improved disease, the regimen was continued for 12 cycles. 
Before the beginning of each 4-week cycle of carboplatin, the absolute neutrophil count needed to be

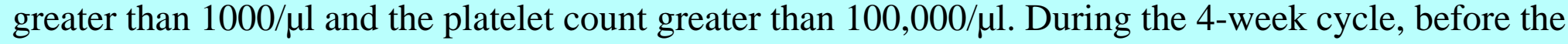
second, third, and fourth dose of carboplatin, the absolute neutrophil count was required to be greater

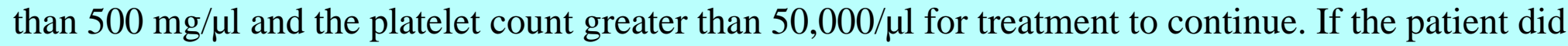
not meet these hematological criteria, treatment was delayed for 1 week. Any patient with fever or neutropenia and/or localized infection had treatment interrupted until these complications resolved. For those patients with more than a 2-week delay in treatment, sepsis associated with neutropenia, or a platelet count less than $20,000 \mu \mathrm{l}$, the next dose of carboplatin was decreased by $50 \%$. For those patients who developed significant vincristine-related neurotoxicity (foot drop, ileus), administration of vincristine was suspended until there was evidence of neurological improvement and the next dose of vincristine was reduced by $50 \%$.

The protocol was approved by the local Institutional Review Board and signed consent was obtained from the parents or legal guardians of the child.

\section{Evaluation of Response}

Neuroradiographic studies were performed before the patient entered the study, after induction chemotherapy, and after every two cycles of maintenance chemotherapy. In addition to the neuroradiographic evaluation, the patients were examined on a weekly basis during induction and maintenance therapy. Responses were based primarily on the contrast-enhanced imaging results and categorized as follows: complete response, the complete disappearance of tumor on imaging studies; partial response, a reduction of $50 \%$ or more in the size of the tumor; minor response, more than $25 \%$ but less than $50 \%$ reduction in tumor size; stable disease, no change or decrease of $25 \%$ or less in tumor size; and progressive disease, any increase in tumor size. The response of the patient was assessed by using the product of the largest two tumor diameters seen on the imaging study. For patients considered to have an objective response (defined as a complete, partial, or minor response), there had to be stable or improved clinical findings associated with a stable or decreased dose of corticosteroid medication. 


\begin{tabular}{|c|c|}
\hline \multicolumn{2}{|c|}{$\begin{array}{c}\text { TABLE } 1 \\
\text { CLINICAL CHARACTERISTICS IN 78 PED LTRIC PATIENTS WITH NEWLY } \\
\text { DIAGNOSED PROGRESSIVE LOW-GRADE GLIOMAS }\end{array}$} \\
\hline Factor & No. of Patierts \\
\hline \multicolumn{2}{|l|}{ age at diagnosis } \\
\hline mean & $3.08 \mathrm{yrs}$ \\
\hline range & $3 \mathrm{mos}-16$ yrs \\
\hline \multicolumn{2}{|l|}{ tumor location } \\
\hline diencephalon & 58 \\
\hline trainstem & 12 \\
\hline cerebral corte $x$ & 2 \\
\hline thalamus & 1 \\
\hline spinal & 1 \\
\hline diffuse leptomeninges & 3 \\
\hline cerebellum & 1 \\
\hline \multicolumn{2}{|l|}{ tumor type } \\
\hline fitrillary & 32 \\
\hline pilocytic & 17 \\
\hline mixedganglia & 3 \\
\hline not determined & 26 \\
\hline yes & 15 \\
\hline no & 63 \\
\hline
\end{tabular}

\section{Patient Population}

Eighty-five children with newly diagnosed, progressive low-grade gliomas were entered into the study. On review, seven of the patients were later found to be ineligible for various reasons, including a greater than 4-week delay from surgery to initiation of chemotherapy (one patient), greater than allowable age at the time of study entry (one patient), parental decision to withdraw the child from the study before initiation of treatment (one patient), ineligible histological diagnosis (one patient), administration of prior chemotherapy (one patient), and delivery of a different chemotherapy regimen (two patients). Clinical characteristics of the patients, including their age at diagnosis, tumor location, signs and symptoms at the time of diagnosis, and histological subtype are given in Tables 1 and 2. Fifteen children met the criteria for a diagnosis of NF-1. The clinical symptoms and signs in patients with and without NF-1 are listed in Table 2. Of the 15 children with NF-1, one who had a diencephalic tumor and in whom a biopsy was obtained prior to entry into the study was found to have pilocytic astrocytoma. Fifty-two of the 63 children without NF-1 had histological confirmation of their diseases, which were pilocytic in 17 , fibrillary in 32, and either a ganglioglioma or mixed glioma in the remaining three patients. All 11 children without NF-1 who were entered into the study without histological confirmation of their disease had diffuse infiltrating diencephalic tumors. 


\begin{tabular}{|c|c|}
\hline \multicolumn{2}{|c|}{$\begin{array}{c}\text { TABLE } 2 \\
\text { SIGNS AND SVMPTOWS AT DIAGNOS IN IN } 78 \text { PED ATRIC PATIENTS WITH NEWLY } \\
\text { DIAGNOSED PROGRESSIVE LOW-GRADE GLIOMAS }\end{array}$} \\
\hline Signs \& Symptoms & No. of Patierts \\
\hline $\begin{array}{l}\text { patients wh NF-1 } \\
\text { decreased wision } \pm \text { nystagm us } \\
\text { nystagm us alone } \\
\text { diencephalic syndrome } \\
\text { macrooephaly } \\
\text { seizures } \\
\text { le thargy' } \\
\text { headache } \\
\text { other } \\
\text { patients w/NF-1 } \\
\text { decreased vision } \pm \text { proptosis } \pm \text { nystagm us } \\
\text { positi se screening tests plus decreased wision } \\
\text { macroophaly } \\
\text { other }\end{array}$ & $\begin{array}{r}24 \\
3 \\
7 \\
10 \\
2 \\
4 \\
5 \\
6\end{array}$ \\
\hline
\end{tabular}

\section{Statistical Considerations}

The length of progression-free survival was measured from the date of patient entry into the study to the date of progressive disease or last contact. Patients who developed allergic or other toxic reactions resulting in discontinuation of treatment were censored at that point. Distributions of progression-free survival and event-free survival were estimated using the technique of Kaplan and Meier[12] and comparisons were made using a Mantel-Haenszel statistic. Standard errors of the Kaplan-Meier estimates were calculated using the technique proposed by Peto and coworkers.[19] The correlation between achieving an objective response and progressive-free survival was evaluated using the landmark method at a landmark of 9 months (time of last best response).[1] Patients who did not show disease progression at 9 months and who were followed for more than 9 months are included in the comparisons of distribution of progression-free survival by response category.

\begin{tabular}{|c|c|c|c|c|c|}
\hline $\begin{array}{l}\text { RAD OGRA PHIC BE } \\
\text { NEWLY }\end{array}$ & SPON & $\begin{array}{l}\text { ABL } \\
\text { TREA } \\
\text { ARES }\end{array}$ & $\begin{array}{l}\text { IN } 78 \\
W-G R\end{array}$ & $\begin{array}{l}\mathrm{RIC} \\
\text { IOM }\end{array}$ & S WITH \\
\hline Tum or Location & $\mathrm{CR}$ & PR & MR & $\mathrm{SD}$ & $\mathrm{PD}$ \\
\hline diencephalic & 2 & 17 & 14 & 22 & 3 \\
\hline trainstem & 1 & 2 & 3 & 4 & 2 \\
\hline cerebral corte $x$ & 0 & 1 & 0 & 1 & 0 \\
\hline le ptomeningeal & 0 & 1 & 1 & 1 & 0 \\
\hline other & 1 & 1 & 0 & 1 & 0 \\
\hline totil & 4 & 22 & 18 & 29 & 5 \\
\hline
\end{tabular}

\section{RESULTS}

\section{Response to Treatment}

The best response to treatment is summarized in Table 3. Forty-four children (56\%) had an objective response to treatment with objective tumor shrinkage. Objective responses were observed not only in patients with diencephalic tumors, but also in patients with tumors of the brainstem, cerebral cortex, and 
leptomeninges. In one-third of the patients, more than $50 \%$ of the tumor regressed while the patient was receiving treatment. Only five patients $(6 \%)$ developed disease progression during the induction treatment. Of the seven children with clinical diencephalic syndrome at the time of diagnosis, two showed a partial response to treatment and three showed a minor response. Of the 11 children who did not have NF-1 or diencephalic tumors and in whom a biopsy was not obtained, five exhibited an objective response to treatment and six showed stable disease. Of the 45 children who responded to treatment, in 22 the maximum response was seen at the end of the induction therapy and in 23 the maximum response was observed at some point during the maintenance treatment.

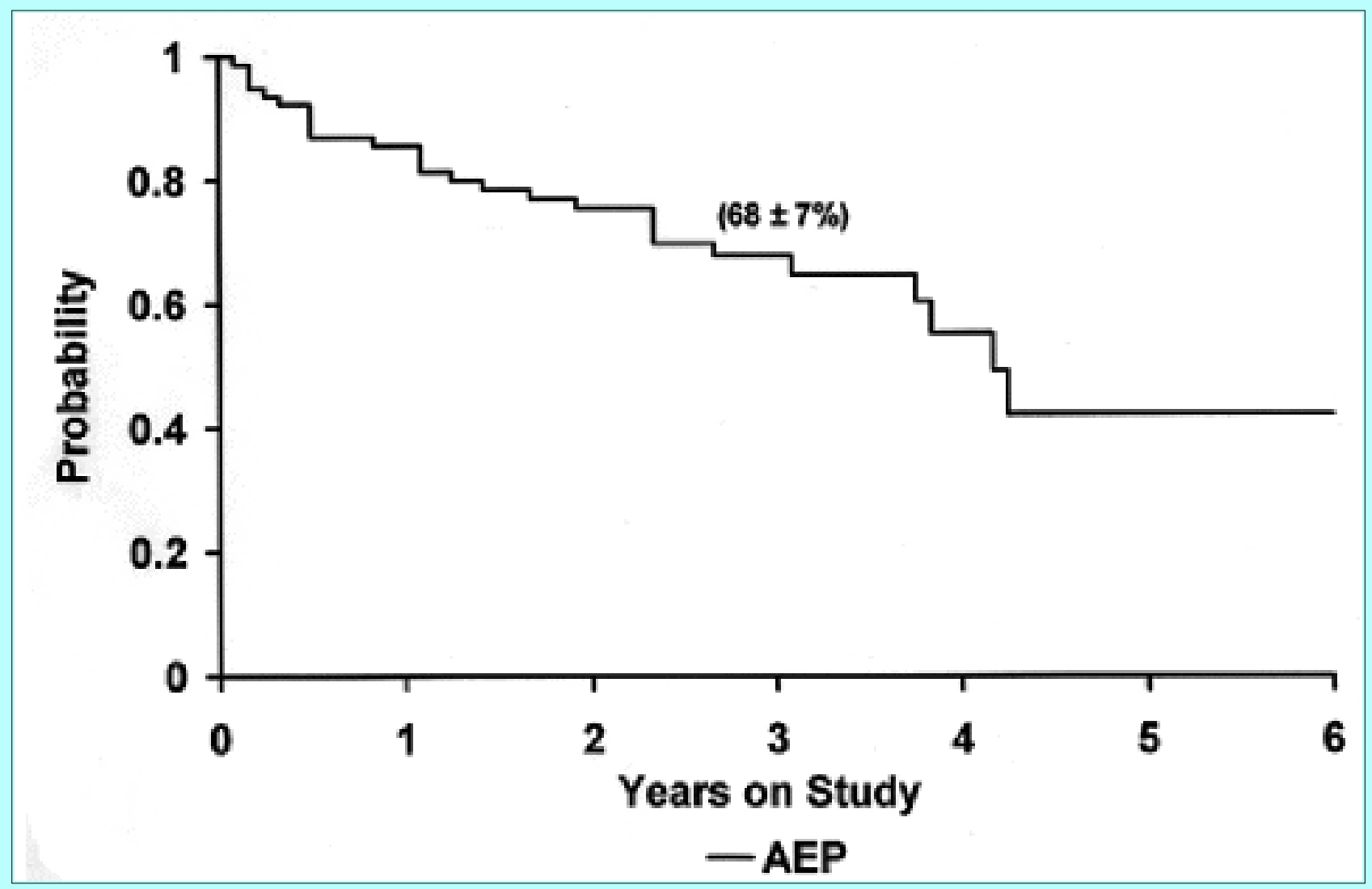

Fig. 1. Graph displaying overall progression-free survival rates in all eligible patients (AEP) from the time they were enrolled in the study (Years on Study).

\section{Progression-Free Survival}

According to the date of their last follow-up examination at the treating institution, the 78 eligible patients enrolled in the study have been followed for a median of 30 months (range 18 months- 6 years) since diagnosis, with 31 children having been followed more than 3 years since diagnosis. The progression-free survival rate at 1 year was $86 \pm 4 \%$, at 2 years $75 \pm 6 \%$, and at 3 years $68 \pm 7 \%$ (Fig. 1). Twenty-seven (35\%) of the 78 patients have developed progressive disease, five during induction therapy, six while on maintenance therapy, and 16 following completion of maintenance therapy. Data in five children were censored from further analysis of progression-free survival when the children developed allergic reactions during maintenance therapy, and one patient died of sepsis after 11 months of treatment. The 45 patients who completed all treatment and have remained progression free since the initiation of the study have been followed for a median of 36 months. Of the five patients who developed allergic reactions, three were switched to immediate local radiotherapy and all remain stable a median of 4 years after the diagnosis; one was given an alternative drug regimen (using etoposide) and remained progression free for 12 months; and one was observed without further treatment and remains stable 48 
months after initiation of the original treatment.

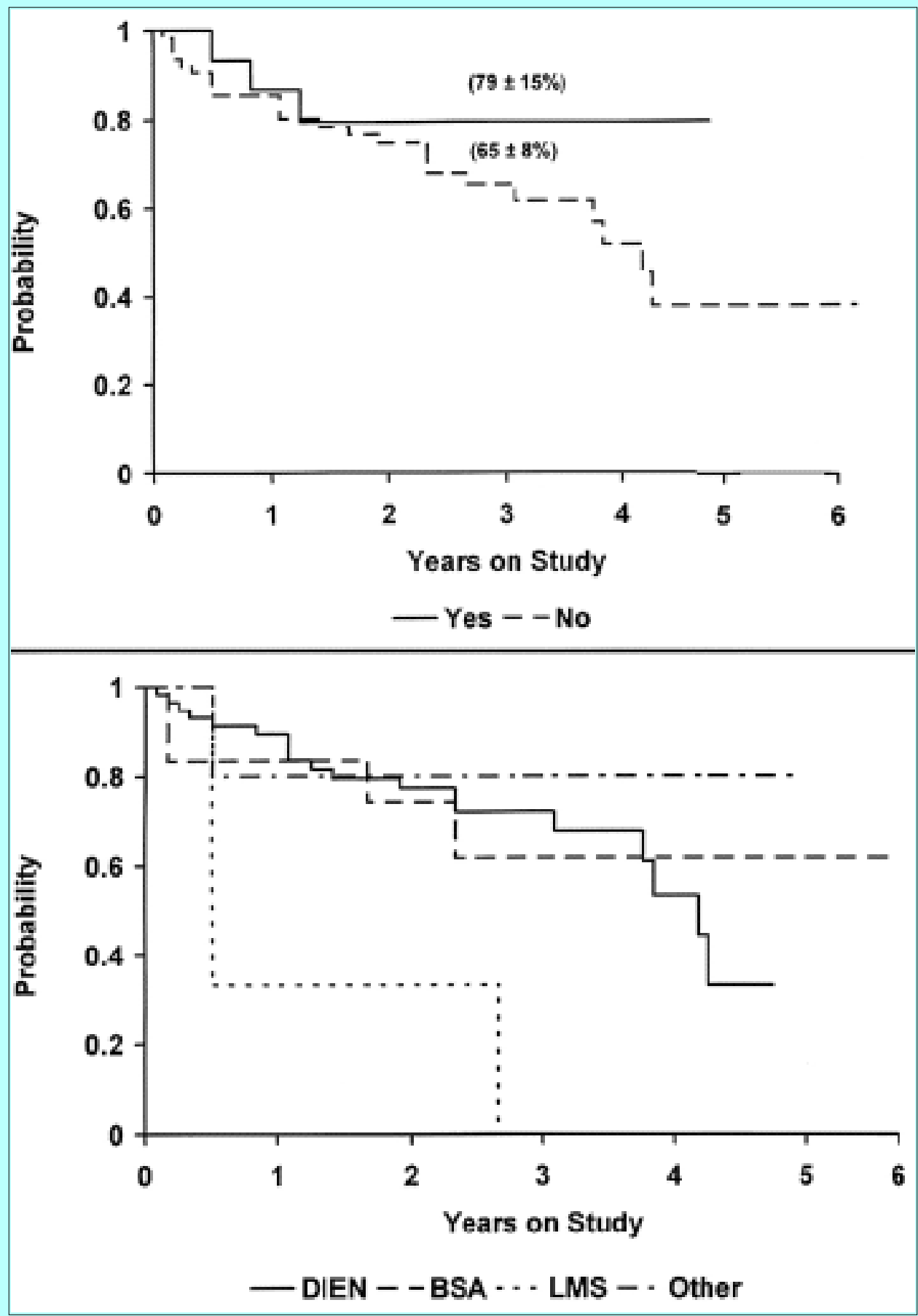

Fig. 2. Graph depicting progression-free survival rates for patients in the study. Upper:

Comparison of patients with NF-1 (Yes) versus those without NF-1 (No). Lower: Comparison of patients with diencephalic (DIEN), brainstem (BSA), leptomeningeal (LMS), and other cranial tumors.

There was no statistical difference between progression-free survival rates in children with or without NF-1: at 2 years those patients with NF-1 had a progression-free survival rate of $79 \pm 11 \%$ compared with $75 \pm 6 \%$ for those without NF-1 (Fig. 2 upper). At 3 years, a comparison of estimated rates of progression-free survival is without merit because only nine children with NF-1 have been followed for more than 3 years. There was no clear-cut statistical difference between the progression-free survival rates in children with diencephalic tumors and those rates in children with brainstem tumors (Fig. 2 
lower). Of the 58 children with diencephalic tumors at the time of diagnosis, the rate of progression-free survival at 3 years was $77 \pm 6 \%$. Seven of those children had presented with the diencephalic syndrome and of these, two have experienced disease progression and five have remained in remission throughout a follow up ranging from 13 to 45 months. Although two of three children with leptomeningeal tumors initially responded to treatment, all developed progressive disease within 3 years of initiation of therapy. Patients with pilocytic astrocytomas (3-year progression-free survival rate $77 \pm 15 \%$ ) did not differ in progression-free survival from those with fibrillary tumors $(63 \pm 11 \%)$ at 2 years of follow up. There was no apparent difference in progression-free survival rates among the different centers at which treatment was given.

There was no correlation between the rate of progression-free survival at 3 years and the best response to therapy when patients who had a complete or partial response ( 25 with a progression-free survival rate of $83 \pm 8 \%$ ) were compared with those who had a minor response or stable disease (38 with a progression-free survival of $73 \pm 9 \%$ ). Similarly, there was no difference in progression-free survival rates when the 43 children who had complete, partial, or minor responses to chemotherapy were compared with the 21 patients with stable disease (3-year progression-free survival $71 \pm 10 \%$ vs. $78 \pm$ $16 \%$, respectively, $\mathrm{p}=0.8$; Fig. 3 upper).

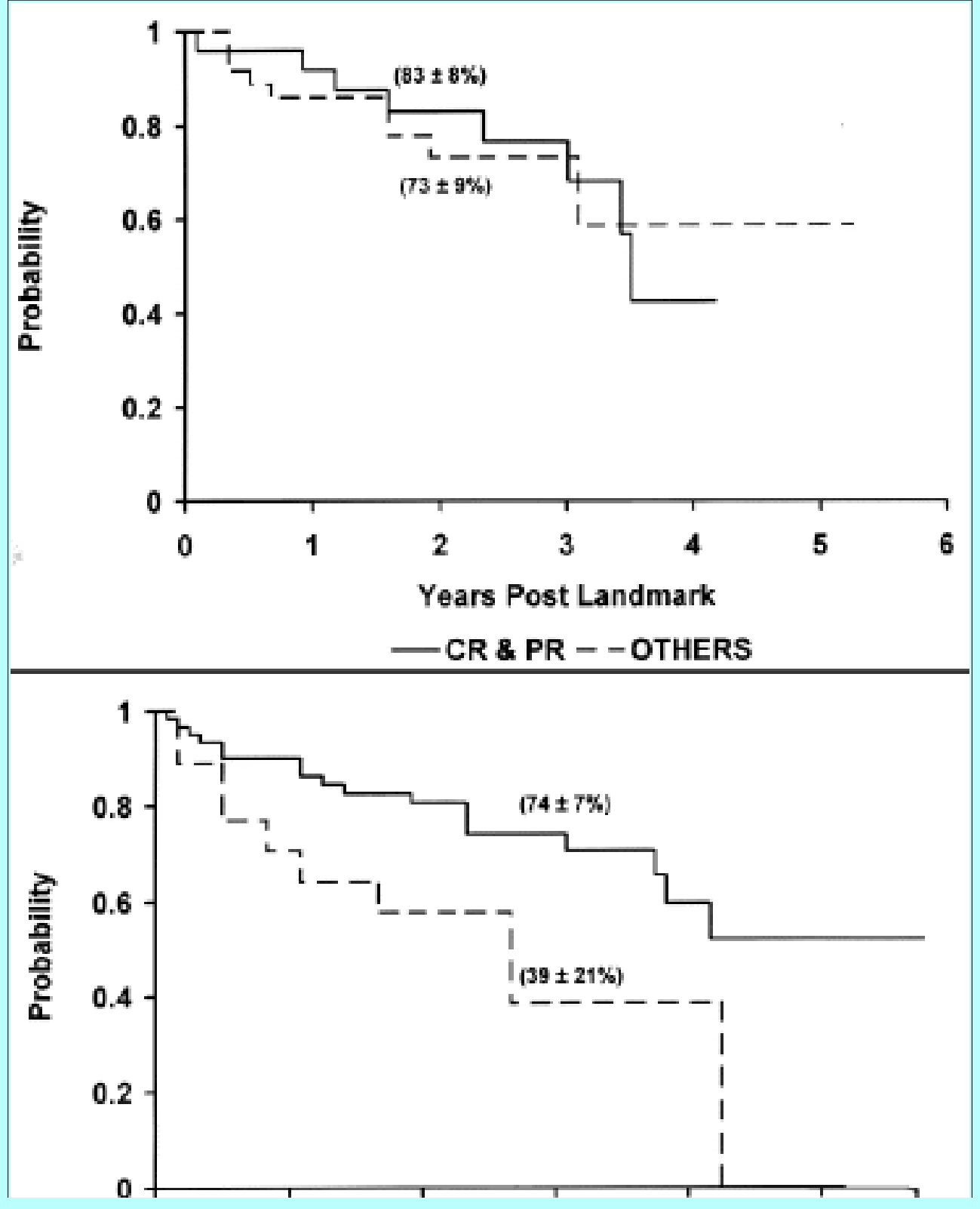




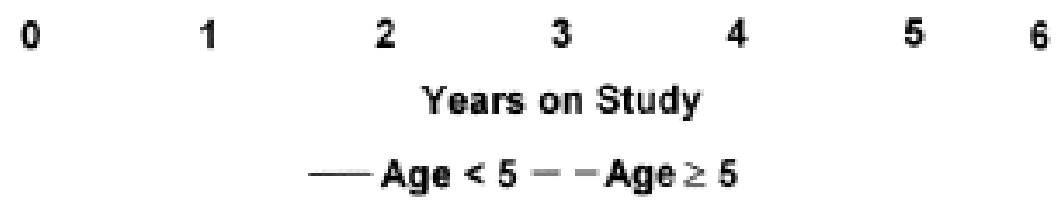

Fig. 3. Graphs displaying the relationships between progression-free survival and response to treatment (upper) and progression-free survival and age in years (lower). $\mathrm{CR}=$ complete response; others $=$ minor response or stable disease; $\mathrm{PR}=$ partial response.

The only statistically significant factor predictive of outcome was age (Fig. 3 lower). The 2-year progression-free survival rate for children 5 years of age or younger at the time treatment began was $81 \pm$ $6 \%$, compared with $58 \pm 14 \%$ in older children $(\mathrm{p}<0.01)$. At 3 years, the progression-free survival rate for children 5 years or younger was $74 \pm 7 \%$ compared with $39 \pm 21 \%$ for older patients $(p<0.01)$.

\section{Toxicity of Treatment}

In general the treatment was relatively well tolerated. No patient was removed from the study because of hematological toxicity, nephrotoxicity, or neurotoxicity, with the exception of the child who died of sepsis after 11 months of treatment. Occasional delays in treatment were caused by prolonged thrombocytopenia or neurotoxicity, predominantly peripheral neuropathy. Five patients were removed from the study because of allergic reactions. All of these occurred during maintenance therapy, between 3 and 8 months after initiation of treatment. One child died of overwhelming sepsis during the eighth course of maintenance chemotherapy, 11 months after diagnosis. In total, 62 children (79\%) completed the prescribed chemotherapy program; the remainder either developed progressive disease (11 patients) or toxicity during treatment.

\section{Overall Survival}

To date, of the 78 patients enrolled in the study, 27 experienced disease progression: 11 during treatment and 16 after completion of the prescribed chemotherapy. Of the 32 patients who were withdrawn from the study because of allergies or who exhibited disease progression while on maintenance, one child died of progressive disease. Including the child who died of sepsis, $76(97 \%)$ of the 78 children treated are alive.

\section{DISCUSSION}

The results in this expanded series of children with newly diagnosed progressive low-grade gliomas, which includes 37 children previously reported, are consistent with the findings of our previously published experience with carboplatin and vincristine for both newly diagnosed and recurrent low-grade gliomas of childhood.[18] In the previous report, 12 of 23 children with recurrent disease had an objective response to the drug regimen, and 23 of 37 newly diagnosed patients also demonstrated objective tumor shrinkage. The $56 \%$ objective response rate seen in the expanded series of 78 children was essentially identical. In addition, as previously noted, the responses were not limited to those children with diencephalic tumors, but were also seen in children with low-grade gliomas of the brainstem and tumors in other sites within the central nervous system. The drug regimen was relatively well tolerated and none of the 78 children had treatment terminated because of hematological renal toxicity or ototoxicity, except for the child who died of sepsis late in maintenance therapy. The use of vincristine was associated with occasional peripheral neuropathy, but this usually responded to a brief interruption or temporary reduction in the vincristine dose. Five children had to be removed from 
treatment because of an allergy to carboplatin. All developed the allergy during maintenance chemotherapy, and this rate of drug allergy is no higher than that noted in the 60 children treated at the time this study was reported in its preliminary form. At the discretion of the treating institution, four of these five patients immediately switched to alternative treatments and could not be assessed for analysis of progression-free survival. No child treated with newly diagnosed disease suffered neurological deterioration thought to be secondary to treatment. In contradistinction, two children with recurrent disease treated during the initial portion of this study suffered neurological deterioration that was followed by clinical improvement after cessation of therapy.[18] It seems most likely that this deterioration seen in patients with recurrent disease was secondary to a radiation therapy recall phenomena, which previously was seen with the use of actinomycin D following radiation in children with cancer. In total, $79 \%$ of the children who began the regimen of carboplatin and vincristine completed all the planned treatment and, excluding the 11 who experienced disease progression while on treatment, $91 \%$ of patients could be treated for the entire course of treatment.

The primary rationale for placing children with residual or progressive low-grade gliomas on a chemotherapy protocol is to delay the need for radiotherapy or other forms of treatment that are potentially damaging, such as aggressive resection. Due to the relatively brief period of follow-up review, we could not address this issue in the preliminary report, although it is clearly the goal of such treatment. Actinomycin-D-vincristine chemotherapy has been used to delay radiotherapy or extensive surgery in children with low-grade gliomas. In a recent update of a study of 29 patients who were treated with the actinomycin-D-vincristine combination and who could be fully evaluated, 12 remained free of subsequent recurrence and radiation therapy was delayed a median of 51 months with more than $70 \%$ of patients not requiring radiation therapy until they were at least 5 years old.[11,17] A five-drug regimen consisting of procarbazine, 6-thioguanine, dibromodulcitol, lomustine, and vincristine was used in 42 children with newly diagnosed symptomatic or previously diagnosed progressive low-grade gliomas by a group at the University of California at San Francisco (R Prados, personal communication, 1996).[20] In this series the mean age of the patients was 5 years and the children had a variety of histological types of low-grade gliomas, similar to those treated in the carboplatin and vincristine series. The median time to treatment failure was 132 weeks and at 5 years the overall survival rate was $78 \%$, with eight children having died of progressive disease.

Carboplatin and iproplatin have been evaluated by the Pediatric Oncology Group to determine both the rate and duration of response.[5] In a series of 12 children treated with carboplatin, stable disease was noted to range between 2 months and 68 months with a median duration of disease control of 40 months. [5] In that series, no objective responses to therapy were noted and children with tumors in all sites of the nervous system were treated. In a separate report, carboplatin alone was noted to control progressive disease for 5 to 32 months in four of six children with newly diagnosed low-grade gliomas.[15] Two patients in this series developed drug hypersensitivity, which resulted in discontinuation of treatment.

Our experience with using carboplatin and vincristine, treating a much larger group of patients than any other study thus far published, suggests that this drug regimen is at least equivalent to, if not better than, other drug regimens in its ability to delay the need for radiotherapy. The progression-free survival rate in the patients treated was $68 \pm 7 \%$ at 3 years; 31 of the patients have been followed more than 3 years since diagnosis without requiring any other form of intervention. Given the small numbers of patients treated in other series; the variability in eligibility, with some studies treating only children with diencephalic tumors and other studies entering patients with tumors in any site in the nervous system; 
and the restriction of some studies to treating only patients less than 5 years of age and others treating children of all ages; no further conclusions can be drawn concerning the superiority of one regimen over another.

It is clear that chemotherapy can delay the need for radiotherapy in children with newly diagnosed, progressive low-grade gliomas. Other approaches may be as effective. There have been reports concerning the use of aggressive surgery to debulk large tumors and treating only patients who have progressive disease after surgical resection.[27] Although this is a reasonable approach for some patients, the surgery itself carries a significant risk of morbidity, especially in young children.[27] In their series of 88 children with biopsy-confirmed low-grade astrocytomas, Hoffman and coworkers[10] suggested that partial resections are often associated with involution of the tumor, yet in their series actual involution was noted in only a few patients. Of note, however, is that partial resections were associated with stable disease in $76 \%$ of patients compared with $67 \%$ of patients whose tumors were less aggressively removed, and that at the time of publication of Hoffman and coworkers' paper, all children with low-grade tumors who had been treated with partial resection were alive at a mean follow-up period of 51 months. Wisoff and associates[27] have reported similar disease control in children with low-grade diencephalic tumors treated by extensive surgical resection. However, very young children, especially those younger than 1 year of age, did poorly after surgery alone. In contrast, in our series, young children, including those younger than 1 year of age, often had an excellent response to chemotherapy. The rationale for using a "conservative" approach in young children consisting of limited initial surgery followed by radiotherapy was strengthened by the recent review by Sutton and coworkers.[25] In their experience with 33 children who would usually have been candidates for initial aggressive resections but were treated initially with less aggressive surgery and either observation or chemotherapy, the overall survival rate at 10.9 years was $85 \%$, with the majority of survivors having useful vision and good academic performance.

Another approach presently being followed is to use more intensive chemotherapy in an attempt to increase overall response and ultimately disease control. Although this seems intuitively reasonable and may prove to be a useful approach, in our experience the degree of response or, for that matter, the determination of an objective response did not correlate with 3-year disease control, as those patients with complete, partial, or minor responses to chemotherapy did not have better 3-year progression-free survival rates than those with stable disease after treatment. This may not be surprising, given the indolent nature of many of these lesions and their slow, partial response to other modalities of treatment, such as radiotherapy. A limitation of this finding is that the neuroradiographic responses of the patients in this study were not centrally reviewed.

Our experience using carboplatin and vincristine demonstrates that children with low-grade tumors outside the diencephalic area will also respond to treatment. The largest subgroup of children, other than those with diencephalic tumors, were those with exophytic, biopsy-confirmed, low-grade brainstem tumors, which were believed not to be amenable to further resection. Excellent responses, including complete responses, were noted in the majority of the 12 children with brainstem tumors who were treated with carboplatin and vincristine. At 2 years, only three of the 12 children with brainstem tumors had developed progressive disease. Also, children who presented with the diencephalic syndrome responded to treatment.

There was no clear impact of the histological type of low-grade glioma on progression-free survival in our series; children with fibrillary and pilocytic astrocytomas were as likely to respond to treatment and 
maintain their response. Similarly, children with NF-1 and those without did not differ in their response to treatment. Despite the frequency with which optic pathway tumors occur in NF-1, only $19 \%$ of patients in this study had NF-1. This probably reflects the requirement of unequivocal evidence for tumor growth prior to study entry. The investigators were hesitant to treat patients with NF-1 unless there was clear-cut evidence of disease progression, given the documented tendency of patients with NF-1 and low-grade tumors to have stable disease for years after initial detection without therapeutic intervention.[16]

The only parameter of prognostic significance was age: older patients were more likely to develop disease progression than younger children. In previous studies, not enough patients of differing ages were included to evaluate this parameter. This finding, if substantiated by other investigators, is of major significance. This lower rate of progression-free survival raises the issue of whether chemotherapy is a useful strategy for older children with low-grade gliomas. Although most would agree that it is worthwhile to delay radiotherapy for 4 or more years in infants with progressive low-grade tumors, the rationale for delaying radiotherapy in older children, especially if only for 1 or 2 years, is less clear. Because many of these older children would be amenable to focal radiotherapy and because limited radiotherapy has never been clearly shown to cause significant sequelae, it is unclear whether the potential side effects of chemotherapy in older children outweigh the benefits of delaying radiotherapy.

\section{CONCLUSIONS}

Our experience in an expanded series of 78 children with progressive low-grade gliomas substantiates the potential efficacy of chemotherapy in at least delaying radiotherapy for the majority of young children with such tumors. This benefit is not only limited to children with diencephalic tumors and is certainly a viable option for patients whose tumors are not amenable to resection without a significant risk of morbidity. The most effective regimen to be used in these patients cannot be gleaned from a single arm study, although the experience with carboplatin and vincristine is encouraging. Prospective randomized studies are required to address the issue of "best" therapy and the relative value of chemotherapy as compared to surgery and/or radiotherapy. The usefulness of chemotherapy for older children with low-grade gliomas, especially for those with limited disease at the time of diagnosis, remains unproven.

\section{References}

1. Alvord EC Jr, Lofton S: Gliomas of the optic nerve or chiasm. Outcome by patients' age, tumor site, and treatment. J Neurosurg 68:85-98, 1988

2. Anderson JR, Cain KC, Gelber RD: Analysis of survival by tumor response. J Clin Oncol 1:710-719, 1983

3. Conway PD, Oechler HW, Kun LE, et al: Importance of histologic condition and treatment of pediatric cerebellar astrocytoma. Cancer 67:2772-2775, 1991

4. Fletcher WA, Imes RK, Hoyt WF: Chiasmal gliomas: appearance and long-term changes demonstrated by computerized tomography. J Neurosurg 65:154-159, 1986

5. Friedman HS, Krischer JP, Burger P, et al: Treatment of children with progressive or recurrent brain tumors with carboplatin or iproplatin: a Pediatric Oncology Group randomized phase II study. J Clin Oncol 10:249-256, 1992 
6. Gilles F: The Childhood Brain Tumor Consortium: a study of childhood brain tumors based on surgical biopsies from ten North American institutions: samples description. J Neurooncol 6:9-23, 1988

7. Gjerris F, Kliken L: Long-term prognosis in children with benign cerebellar astrocytoma. $\mathbf{J}$ Neurosurg 49:179-184, 1978

8. Heideman RL, Packer RJ, Albright LA, et al: Tumors of the central nervous system, in Pizzo PA, Poplack DG (eds): Principles and Practice of Pediatric Oncology. Philadelphia: Lippincott, 1989, pp $505-544$

9. Hirsch JF, Sainte Rose C, Pierre-Kahn A, et al: Benign astrocytic and oligodendrocytic tumors of the cerebral hemispheres in children. J Neurosurg 70:568-572, 1989

10. Hoffman HJ, Soloniuk DS, Humphreys RP, et al: Management and outcome of low-grade astrocytomas of the midline in children: a retrospective review. Neurosurgery 33:964-971, 1993

11. Janss AJ, Grundy R, Cnaan A, et al: Optic pathway and hypothalamic/chiasmatic gliomas in children younger than age 5 years with a 6-year follow-up. Cancer 75:1051-1059, 1995

12. Kaplan EL, Meier P: Nonparametric estimation from incomplete observations. J Am Stat Assoc 53:457-481, 1958

13. Kleihues P, Burger PC, Scheithauer BW: Histologic Typing of Tumours of the Central Nervous System. Berlin: Springer-Verlag, 1993

14. Lefkowitz IB, Packer RJ, Sutton LN, et al: Results of the treatment of children with recurrent gliomas with lomustine and vincristine. Cancer 61:896-902, 1988

15. Moghrabi A, Friedman HS, Burger PC, et al: Carboplatin treatment of progressive optic pathway gliomas to delay radiotherapy. J Neurosurg 79:223-227, 1993

16. Packer RJ, Bilaniuk LT, Cohen BH, et al: Intracranial visual pathway gliomas in children with neurofibromatosis. Neurofibromatosis 1:212-222, 1988

17. Packer RJ, Lange B, Ater J, et al: Carboplatin and vincristine for recurrent and newly diagnosed low-grade gliomas of childhood. J Clin Oncol 11:850-856, 1993

18. Packer RJ, Sutton LM, Bilaniuk LT, et al: Treatment of chiasmatic/hypothalamic gliomas of childhood with chemotherapy: an update. Ann Neurol 23:79-85, 1988

19. Peto R, Pike MC, Armitage P, et al: Design and analysis of randomised clinical trials requiring prolonged observation of each patient. I. Introduction and design. Br J Cancer 34:585-612, 1976

20. Petronio J, Edwards MSB, Prados M, et al: Management of chiasmal and hypothalamic gliomas of infancy and childhood with chemotherapy. J Neurosurg 74:701-708, 1991

21. Pons MA, Finlay JL, Walker RW, et al: Chemotherapy with vincristine (VCR) and etoposide (VP-16) in children with low-grade astrocytoma. J Neurooncol 14:151-158, 1992

22. Rush JA, Young BR, Campbell RJ, et al: Optic glioma. Long-term follow-up of 85 histopathologically verified cases. Ophthalmology 89:1213-1219, 1982 
23. Shaw EG, Scheithauer BW, Gilbertson DT, et al: Post-operative radiotherapy of supratentorial low-grade gliomas. Int J Radiat Oncol Biol Phys 16:663-668, 1989

24. Sumner T, Freeman AI, Cohen M, et al: Chemotherapy in recurrent noncystic low-grade astrocytomas of the cerebrum in children. J Surg Oncol 10:45-54, 1978

25. Sutton LN, Molloy PT, Sernyak H, et al: Long-term outcome of hypothalamic/chiasmatic astrocytomas in children treated with conservative surgery. J Neurosurg 83:583-589, 1995

26. Taveras JM, Mont LA, Wood EH: The value of radiation therapy in the management of gliomas of the optic nerves and chiasm. Radiology 66:518-528, 1956

27. Wisoff JH, Abbott R, Epstein F: Surgical management of exophytic chiasmatic-hypothalamic tumors of childhood. J Neurosurg 73:661-667, 1990

Manuscript received April 30, 1996.

Accepted in final form January 7, 1997.

Address reprint requests to: Roger J. Packer, M.D., Department of Neurology, Children's National Medical Center, 111 Michigan Avenue, NW, Washington, D.C. 20010. 